\title{
Deduction of Continuum Potentials from Planar Channeling
}

\section{Citation}

Gibson, W. M., and J. Golovchenko. 1972. Deduction of Continuum Potentials from Planar Channeling. Physical Review Letters. doi:10.1103/physrevlett.28.1301.

\section{Published Version}

doi:10.1103/PhysRevLett.28.1301

\section{Permanent link}

http://nrs.harvard.edu/urn-3:HUL.InstRepos:29407071

\section{Terms of Use}

This article was downloaded from Harvard University's DASH repository, and is made available under the terms and conditions applicable to Other Posted Material, as set forth at http:// nrs.harvard.edu/urn-3:HUL.InstRepos:dash.current.terms-of-use\#LAA

\section{Share Your Story}

The Harvard community has made this article openly available.

Please share how this access benefits you. Submit a story.

\section{Accessibility}


Ledingham, Norelco Rep. 25, 112 (1967).

${ }^{7}$ J. A. Bearden, Rev. Mod. Phys. 39, 78 (1967).

${ }^{8} \mathrm{H}$. W. Lewis, B. E. Simmons, and E. Merzbacher, Phys. Rev. 91, 943 (1953).

${ }^{9}$ A derivation of Eq. (4), enumerating all approximations, is given in J. Macek, unpublished report available from the author.

${ }^{10}$ D. H. Jaecks, Ph.D. thesis, University of Washing- ton, 1964 (unpublished); see also C. E. Head and R. H. Hughes, Phys. Rev. 138, A1630 (1965).

${ }^{11}$ E. J. McGuire, Phys. Rev. A 3, 587 (1970).

${ }^{12}$ W. S. Johnson and J. E. Gibbons, Projected Range Statistics in Semiconductors (Stanford Univ. Bookshop, Stanford, Calif., 1970).

${ }^{13}$ D. L. Walters and C. P. Bhalla, Phys. Rev. A $\underline{3}$, 1919 (1971).

\title{
Deduction of Continuum Potentials from Planar Channeling
}

\author{
W. M. Gibson \\ Bell Laboratories, Murray Hill, New Jersey 07974 \\ and \\ J. Golovchenko* \\ Rensselaer Polytechnic Institute, Troy, New York 12181, and Bell Laboratories, Murray Hill, New Jersey 07974
}

(Received 24 January 1972)

\begin{abstract}
A new method for determining planar continuum potentials from planar channeling data has been developed. Preliminary experiments have been done for $1.8-\mathrm{MeV} \alpha$ particles in [111] gold planar directions from which the continuum potential has been deduced.
\end{abstract}

During the past several years the experimental observation of fine structure in the energy loss of planar-channeled particles having specific trajectories in transmission through thin crystals has been exploited by several authors ${ }^{1-7}$ in an attempt to characterize the detailed motion of the penetrating particle and associated phenomena. Of particular importance in such studies is the planar continuum potential ${ }^{8.9}$ since this function serves as the starting point for more detailed experimental and theoretical investigations of channeled particle properties. In this Letter we report on a new and direct method of obtaining the continuum potential from the analysis of planar channeling data.

This approach to determing the continuum potential is based upon the realization that for a symmetric one-dimensional anharmonic oscillator of known mass, knowledge of the oscillation period as a function of the total oscillator energy is sufficient to uniquely deduce the potential function responsible for the motion. ${ }^{10}$ The pioneering work of Datz et al. ${ }^{3}$ showed how the wavelengths of the transverse motion of planar-channeled particles can be determined in a straightforward way, from which the period of the transverse oscillations follows directly. In this work we show a direct method to extract the energy of the transverse motion, which together with the wavelength measurement provides enough information to uniquely determine the continuum potential necessary to describe the transverse motion of the channeled particle.

Briefly reviewing the experimental procedure, after the planar-channeled beam emerges from a thin crystal, it is energy analyzed with a masked solid-state detector having an angular resolution of $\sim 2.5 \times 10^{-7} \mathrm{sr}$, positioned in line with the incident beam. Under these conditions the particles recorded by the detector are mainly those whose trajectories have undergone an integral number of wavelengths in the crystal. ${ }^{3}$ If the detector has sufficient energy resolution, particles having different numbers of wavelengths in the crystal can be distinguished since each has a characteristic energy loss rate, corresponding to different amplitudes of oscillation in the channel. A schematic illustration of the trajectory selection principle is shown in Fig. 1. (As can be seen from this figure, the wavelength of the motion of a particular group can be determined by experimentally increasing the crystal thickness $L$ until another group of an equal energy loss rate is found. The increase in thickness will be 1 wavelength for those groups. ${ }^{3}$ )

For this detector geometry, changing the angle between the crystal plane and the incident beam, keeping the crystal thickness along the beam direction constant, has the effect of changing the populations of the various detected wavelength 


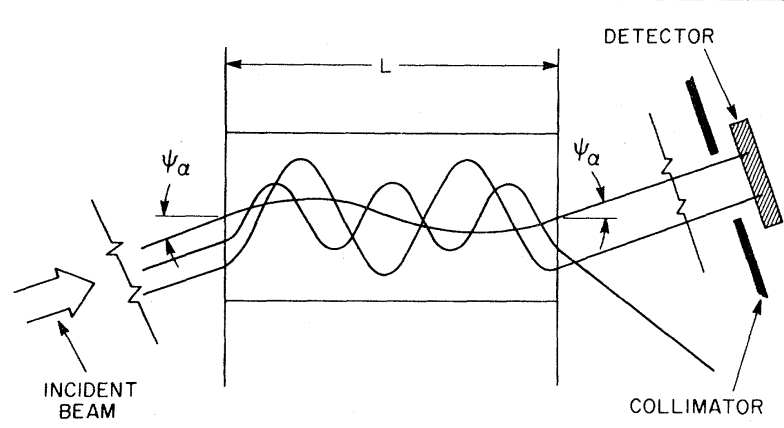

FIG. 1. Possible trajectories of planar-channeled particles. The two trajectories shown entering the detector satisfy the boundary conditions that exit and entrance angles at the crystal boundary are equal.

groups. A calculation of group population as a function of $\psi_{\alpha}$, the angle between the incident beam and the plane, indicates that for a perfect crystal $^{11}$

$$
n_{j} \propto\left(d \bar{V} /\left.d y\right|_{y_{0 j}\left(\psi_{\alpha}\right)}\right)^{-2},
$$

where $n_{j}$ is the population of the $j$ th group, $y$ is the distance from the midplane position, and $\bar{V}$ is the planar continuum potential. The derivative is to be evaluated at the point $y_{j}$ at which the particles in the $j$ th group enter the channel. As $\psi_{\alpha}$ increases from zero, $y_{0}$ decreases from $A_{j}$, the amplitude of the group, to zero. At $y_{0 j}$ equal to zero, the slope of the symmetric potential must be zero, and $n_{j}$ goes through a strong maximum at the corresponding $\psi_{\alpha}=\psi_{\alpha \max }$. For $\psi_{\alpha}>\psi_{\alpha \max }$ in a perfect crystal the group can no longer be populated. Experimental measurements of group population as a function of incidence angle, as shown in Fig. 2, fit this description except for smoothing of the expected sharp angular dependence in the region of large $n_{j}$, due to mosaic spread, and multiple scattering. When the population of a particular group is maximized, entry into the crystal for those particles occurs at midchannel, i.e., $y_{0 j}=0$, and, satisfying the condition of integral number of wavelengths in the crystal, the particles also exit at $y_{0 j}=0$, where the transverse kinetic energy is a maximum. The transverse energy $E_{\perp}$ inside the crystal is given by

$$
E_{\perp}=E \psi_{\alpha}^{2}+V\left(y_{0}\right)
$$

where $E$ is the beam energy, and $\psi_{\alpha}$ is assumed small. Thus, referring energy to the potential of $y=0$,

$$
E_{\perp}=E \psi_{\alpha j}{ }^{2},
$$

where $\psi_{\alpha j}$ is the angle that maximizes the group

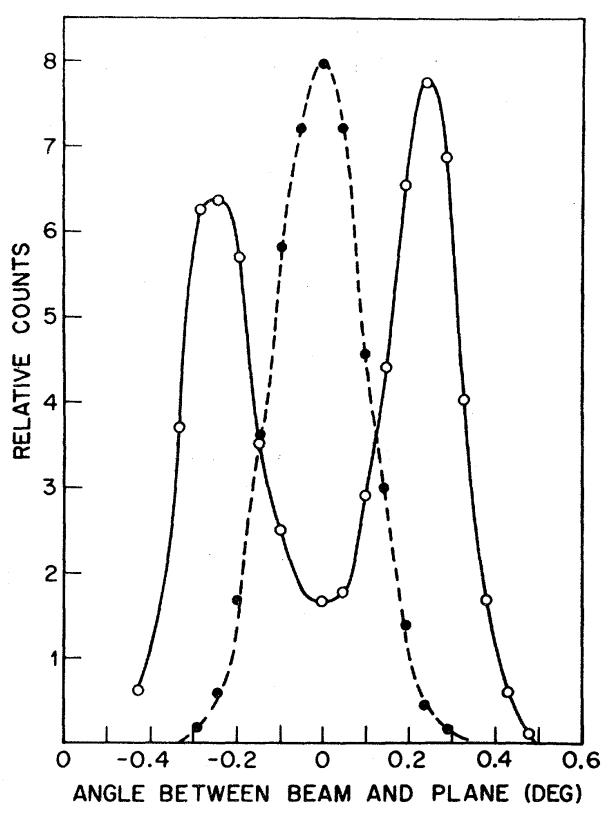

FIG. 2. An angular scan (solid curve) of a group having three complete oscillations in the [111] plane of a gold sample tilted to a thickness of $2750 \AA$. The incident beam consisted of $1.8 \mathrm{-MeV} \alpha$ particles. Also shown (dashed curve) is the least-energy-loss group whose transverse energies are so small that a continuum of wavelengths is detected about the zero-transverse-energy wavelength. The peak population of this group serves as an angular reference. The scales for the two plots differ by a factor of 3 , with the central group being the more intense.

population.

With these techniques one can obtain the wavelength of oscillation as a function of the transverse energy. By changing the thickness of the crystal along the beam direction, one changes the wavelengths of the detected groups, and thus each different thickness measurement yields additional evaluation points for the desired wavelength-transverse energy function.

The method of extracting the potential function proceeds as follows. A wavelength function $\lambda(E$, $V)$ is chosen that fits the data best in a leastsquares sense. $\lambda$ is to be considered a function of $E$ and a functional of the transverse continuum potential. The dependence of $\lambda$ upon the potential function is represented by the parameters $a_{1}, a_{2}$, $\cdots, a_{n}$ [i.e., $\lambda\left(E, a_{1}, a_{2}, \cdots, a_{n}\right)$ ], where the $a$ 's are chosen by the least-squares criterion. (The choice of $\lambda$ requires more detailed discussion to which we will return shortly.) One can then obtain the inverse of the potential via the transformation $^{10}$

$$
y(V)=\left(8 \pi^{2} M v_{F}^{2}\right)^{-1 / 2} \int_{0}^{V} \lambda\left(E_{\perp}\right)\left(V-E_{\perp}\right)^{-1 / 2} d E_{\perp},
$$


where $v_{F}$ is the velocity of the beam along the channel direction.

For $\lambda\left(E_{\perp}, a\right)$ it is desirable to choose a function that fits the data with the least number of adjustable parameters and represents a physically reasonable potential whose parameters can be given some easily interpretable physical significance.

For the present analysis we have used

$$
\lambda=\lambda_{0}(2 / \pi)(1+4 \Gamma B)^{-1 / 4} K\left[\frac{1}{2}-\frac{1}{2}(1+4 \Gamma B)^{-1 / 2}\right],
$$

with $\lambda_{0}=2 \pi v_{F}\left(M / a_{1}\right)^{1 / 2}, \Gamma=E_{T} / E_{\max }, B=E_{\max } a_{2} / a_{1}^{2}$, $K$ the complete elliptic integral of the first kind (see Milne-Thompson ${ }^{12}$ ), and $E_{\max }$ only inserted for normalization purposes. Transformation of this function results in a potential function,

$$
V=\frac{1}{2} a_{1} y^{2}+\frac{1}{4} a_{2} y^{4} \text {. }
$$

Equation (2) is more easily obtained from Eq. (3) than vice versa.

This $\lambda$ function is attractive since the parameters $a_{1}, a_{2}$ have a simple physical significance in the potential function which is of a fairly general form. (The next term to appear in the powerseries expansion would be $a_{3} y^{6}$ since $V$ is symmetric about $y=0$.) In the final analysis we can judge how good a choice of $\lambda$ we have made by examining the fit to the data.

Plotted in Fig. 3(a) is $\lambda / \lambda_{0}$ of Eq. (2) versus $\Gamma=E_{\perp} / E_{\max }$ for different values of $B=E_{\max } a_{2} / a_{1}{ }^{2}$. $E_{\text {max }}$ is arbitrary and for convenience chosen as the maximum observed transverse energy. In Fig. 3(a) we also show experimental results for 1.8-MeV $\alpha$ particles channeled through a 2500gold foil along the [111] plane. These results are well reproduced by the fitting function Eq. (2) for $\lambda_{0}=1408 \AA$ and $E_{\max } a_{2} / a_{1}{ }^{2}=3.61$ with $E_{\max }=103.5$ as shown by the solid curve of Fig. 3(a). The resulting coefficients of the potential are $a_{1}=71.6$ \pm 4.6 and $a_{2}=179 \pm 26$. The uncertainties are conservative estimates based upon experimental uncertainty in the crystal thickness and the transverse energy determinations. The value of $a_{1}$ can be compared with a value of 75 obtained from the curvature parameter deduced by Robinson ${ }^{7}$ from energy loss measurements and an empirical relationship between the frequency and energy loss. The measured potential distribution is shown in Fig. 3(b) and is compared with the planar potential derived from the Moliere approximation to the Thomas-Fermi interatomic potential. ${ }^{8}$

We would like to emphasize at this point that the method presented in this work represents a deduction of the potential function that is consistent with the original model $^{3}$ for the explanation
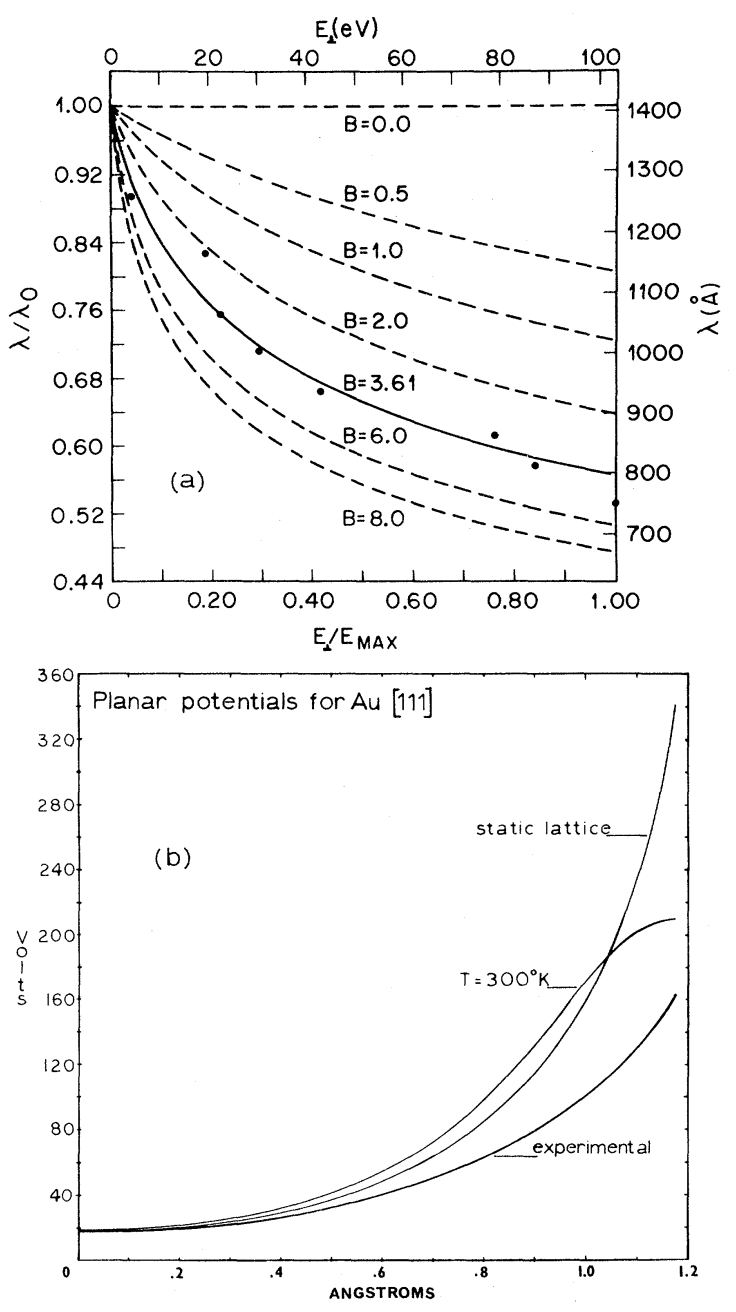

FIG. 3. (a) Values of $\lambda / \lambda_{0}$ versus $E_{\perp} / E_{\max }$ for various values of $B$ are shown in the solid and dashed curves, which are read on the left-hand and bottom axes. The data and fit by the solid curve are read on the righthand and top axes. $B=0$ corresponds to a harmonic oscillator potential, where the wavelength is independent of transverse energy. The data are for $1.8-\mathrm{MeV} \alpha$ particles in [111] Au channels. (b) The interplanar continuum potential for [111] planes in gold obtained from the fit shown in (a). The experimental value of the potential is normalized at $y=0$ to theoretical interplanar potentials calculated from a Thomas-Fermi interatomic potential, which are shown for comparison.

of group structure in energy loss experiments of this type. This approach to determining the potential function has the advantage of not relying on any assumptions about stopping power, as have been used in previous analyses (see Refs. 4 and 7), and thus gives the study of the motion of channeled particles a more fundamental foundation upon which discussion of other properties of channeled particles (stopping power, multiple 
scattering, flux peaking, etc.) can be built.

We wish to acknowledge helpful discussions with J. Bøttiger and W. L。 Brown, and experimental assistance from $R$. Levesque. The thin gold crystal used in the measurements was kindly supplied by P. Ambrosius-Olson of Aarhus University.

*Work supported in part by a U. S. Atomic Energy Commission Graduate Fellowship.

${ }^{1}$ H. O. Lutz, S. Datz, C. D. Moak, and T. S. Noggle, Phys. Rev. Lett. 17, 6 (1966).

${ }^{2}$ W. M. Gibson, J. B. Rasmussen, P. Ambrosius-Olsen, and C. J. Andreen, Can. J. Phys. 46, 551 (1968).

${ }^{3}$ S. Datz, C. D. Moak, T. S. Noggle, B. R. Appleton, and $\mathrm{H}_{\circ}$ O. Lutz, Phys. Rev. 179, 315 (1969).

${ }^{4}$ M. T. Robinson, Phys. Rev. 179, 327 (1969).

${ }^{5}$ B. R. Appleton, S. Datz, C. D. Moak, and M. T. Robinson, Phys. Rev. B 4, 1452 (1971).

${ }^{6}$ F. H. Eisen and M. T. Robinson, Phys. Rev. B $\underline{4}$, 1457 (1971).

${ }^{7}$ M. T. Robinson, Phys. Rev. B 4, 1461 (1971).

${ }^{8}$ C. Erginsoy, Phys. Rev. Lett. 15, 360 (1965).

${ }^{9} \mathrm{~J}$. Lindhard, Kgl. Dan. Vidensk. Selsk., Mat.-Fys. Medd. 34, No. 14 (1965).

${ }^{10}$ L. D. Landau and E. M. Lifshitz, Mechanics (Pergamon, New York, 1960), p. 27.

${ }^{11}$ W. M. Gibson and J. Golovchenko, to be published.

${ }^{12}$ L. M. Milne-Thompson, in Handbook of Mathematical Functions, edited by M. Abramowitz and I. A. Stegun (U.S. GPO, Washington, D. C., 1964), p. 589.

\title{
Ultrasonic Spin Echoes
}

\author{
N. S. Shiren and T. G. Kazyaka \\ IBM Thomas J. Watson Research Center, Yorktown Heights, New York 10598
}

(Received 24 March 1972)

\begin{abstract}
Generation and detection of ultrasonic spin echoes (phonon echoes) and of ultrasonically induced rf magnetic field spin echoes are reported. With the latter, signal-to-noise improvements of 2 orders of magnitude were found over direct ultrasonic absorption spectroscopy. The effects of momentum conservation selection rules are discussed, as are properties and applications of the various echoes.
\end{abstract}

We report the first observations of ultrasonic spin echoes (or phonon echoes), and of ultrasonically induced $\mathrm{rf}$ magnetic spin echoes. The ultrasonic spin echoes are generated following twopulse sequences which consist of either two ultrasonic pulses ( $U U$ echo), or an ultrasonic pulse followed by an $\mathrm{rf}$ magnetic field pulse ( $U H$ echo). Ultrasonically induced rf spin echoes are generated by three-pulse sequences consisting of two ultrasonic pulses and an rf magnetic field pulse in any order (UUH, UHU, HUU echoes); these are stimulated echoes. ${ }^{1}$ As discussed below, the form an echo takes (i.e., whether ultrasonic or magnetic), and its propagation direction, are determined by momentum conservation selection rules. $^{2} U U$ echoes are analogous to photon echoes, ${ }^{3}$ and propagate in the forward direction (with respect to the ultrasonic pulses); $U H$ echoes propagate in the backward direction. Ultrasonic spin echoes are of interest because their temporal decay measures the relaxation of off-diagonal components of magnetoelastic stress, whereas the decay of magnetic $(\mathrm{HH})$ spin echoes ${ }^{1}$ measures the relaxation of the off-diagonal magnetization. The relaxation time constants are not equal when the dominant spin-phonon coupling is quadratic in the spin operators, as is usual for
non-Kramers ions. Therefore $U U$ or $U H$ echoes complement $H H$ echoes in obtaining a complete description of paramagnetic relaxation processes. They should also be of use in the measurement of spin-phonon coupling constants. We believe the stimulated echoes can make ultrasonic paramagnetic resonance a more important adjunct to other EPR spectroscopic techniques than it has been heretofore, since we have observed signal-tonoise improvements of 2 orders of magnitude over direct ultrasonic absorption measurements on weakly coupled ions. In addition, they can be observed simultaneously with other EPR measurements in the same spectrometer.

The experiments were performed at $9 \mathrm{GHz}$ on paramagnetic ions in concentrations $\approx 10^{-5}$ in $\mathrm{MgO}$ crystals at 1.8 and $4.2^{\circ} \mathrm{K}$. The measurements reported here utilize $1.75-\mathrm{cm}$-long quartz transducers bonded to $\mathrm{MgO}$ crystals $\approx 1.25 \mathrm{~cm}$ in length, although our first observations were made using thin-film $\mathrm{ZnO}$ transducers deposited directly on the $\mathrm{MgO}$. The ultrasonic measurements were of the reflection type; i.e., the generating cavity resonator (with the usual re-entrant configuration) also served as detector. rf magnetic field pulses were applied via a rectangular, dielectric filled, $\mathrm{TE}_{012}$ cavity resonator 